\title{
Algoritmos FPT para reconhecer grafos bem cobertos
}

\author{
Rafael T. Araújo ${ }^{1}$, Sulamita Klein ${ }^{2}$, Rudini Sampaio ${ }^{1}$ \\ ${ }^{1}$ Universidade Federal do Ceará, Fortaleza, Brazil \\ ${ }^{2}$ Universidade Federal do Rio de Janeiro, Rio de Janeiro, Brazil \\ \{rafaelteixeira, rudini\}@lia.ufc.br, sula@cos.ufrj.br
}

\begin{abstract}
Resumo. Dado um grafo $G$, sejam vc $(G)$ e $v c^{+}(G)$ os tamanhos de uma cobertura mínima de vértices e de uma máxima cobertura minimal de vértices, respectivamente. Dizemos que $G$ é bem coberto se $v c(G)=v c^{+}(G)$ (ou seja, todas as coberturas minimais são mínimas). É coNP-completo decidir se um grafo é bem coberto. Nesse artigo, obtemos algoritmos FPT de tempos $O^{*}\left(2^{v c}\right)$ e $O^{*}\left(1.4656^{v c^{+}}\right)$para decidir se um grafo é bem coberto, parametrizados por vc $(G)$ e $v c^{+}(G)$, respectivamente, melhorando resultados de Boria et al. em 2015. Também obtemos algoritmo FPT parametrizado por $\alpha(G)=$ $n-v c(G)$ em grafos d-degenerados, que inclui grafos com genus limitado (como grafos planares) e grafos com grau máximo limitado. Finalmente usamos a decomposição primeval para obter algoritmo linear para grafos $P_{4}$-laden estendidos e grafos $(q, q-4)$, que é FPT parametrizado por $q$, melhorando resultados de Klein et al. em 2013.
\end{abstract}

\section{Introdução}

Seja $G=(V, E)$ um grafo e $C, I \subseteq V$. Dizemos que $C$ é cobertura se toda aresta de $G$ tem extremidade em $C$ e que $I$ é independente se todos os vértices em $I$ são não adjacentes entre si. Sabe-se que $C$ é cobertura se e somente se $V-C$ é independente. Seja $v c(G)$ o tamanho de uma cobertura mínima e $\alpha(G)=n-v c(G)$ o tamanho do maior conjunto independente de $G$. Um grafo é bem coberto se todas coberturas minimais são mínimas (ou também se todos conjuntos independentes maximais são máximos). Grafos bem cobertos foram introduzidos em 1970 por [Plummer 1970]. São interessantes pois o algoritmo guloso que gera um conjunto independente maximal sempre gera um conjunto independente máximo (e também uma cobertura mínima). Porém, decidir se um grafo é bem coberto é coNP-completo mesmo em grafos livres de $K_{1,4}$ [Caro et al. 1996].

Nesse artigo, investigamos esse problema com relação à sua complexidade parametrizada e obtemos algoritmos FPT de tempos $O^{*}\left(2^{v c}\right)$ e $O^{*}\left(1.4656^{v c^{+}}\right)$para decidir se um grafo é bem coberto, parametrizados por $v c(G)$ e $v c^{+}(G)$, respectivamente, melhorando resultados de Boria et al. em 2015 [Boria et al. 2015]. Também obtemos algoritmo FPT parametrizado por $\alpha(G)=n-v c(G)$ em grafos $d$-degenerados, que inclui grafos com genus limitado (como grafos planares) e grafos com grau máximo limitado.

Finalmente estudamos a boa cobertura de grafos com poucos $P_{4}$ 's. Em [Klein et al. 2013], a boa cobertura de muitas classes de grafos com poucos $P_{4}$ 's foi investigada, como os cografos, $P_{4}$-esparsos, $P_{4}$-lite e $P_{4}$-tidy. Nesse artigo, também obtemos algoritmo linear para grafos $P_{4}$-laden estendidos e grafos $(q, q-4)$, que são superclasses dessas classes (ver Figura 1). Esse algoritmo é FPT parametrizado por $q$. 


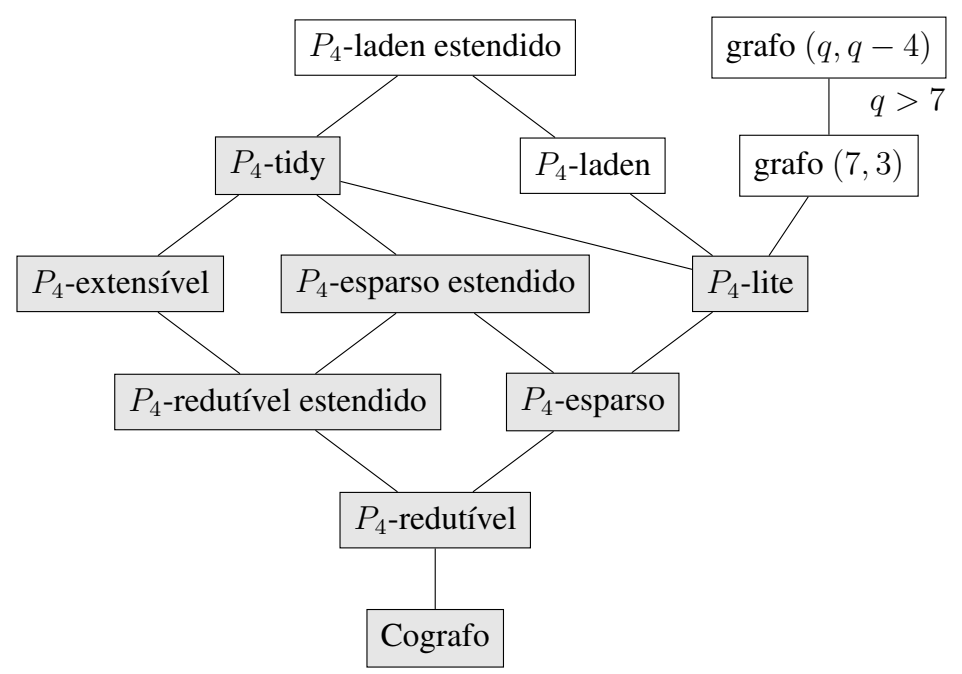

Figure 1. Hierarquia das classes investigadas. Em cinza, as classes investigadas em [Klein et al. 2013].

\section{Boa cobertura de grafos com poucos $\boldsymbol{P}_{4}$ 's}

Cografo é um grafo sem $P_{4}$ induzido. Um grafo é $P_{4}$-esparso se todo grupo de 5 vértices induz no máximo um $P_{4}$. Dado $q \geq 4$, um grafo é $(q, q-4)$ se todo grupo de no máximo $q$ vértices induz no máximo $q-4 P_{4}$ 's. Cografos e $P_{4}$-esparsos são exatamente os grafos $(4,0)$ e $(5,1)$. Em [Babel et al. 2001], foram obtidos algoritmos lineares para vários problemas de otimização em grafos $(q, q-4)$. Um grafo é $P_{4}$-laden estendido se todo subgrafo induzido de no máximo 6 vértices é livre de $\left\{2 K_{2}, C_{4}\right\}$ ou contém dois $P_{4}$ 's induzidos [Giakoumakis 1996]. Uma motivação para obter algoritmos em $P_{4}$-laden estendidos e grafos $(q, q-4)$ é por eles estarem no topo de uma hierarquia bem conhecida de grafos com poucos $P_{4}$ 's, como $P_{4}$-lite, $P_{4}$-laden e $P_{4}$-tidy (Figura 1). Em [Klein et al. 2013], foram obtidos algoritmos lineares para reconhecer grafos $P_{4}$-tidy bem cobertos. Usando a decomposição primeval dos $P_{4}$-laden estendidos e dos grafos $(q, q-4)$, obtemos:

Teorema 1 Seja $G$ um grafo e $q \geq 4$. Se $G$ é $(q, q-4)$ ou $P_{4}$-laden estendido, é possível decidir se $G$ é bem coberto em tempo linear $O\left(2^{q} q^{2} \cdot(m+n)\right)$.

\section{FPT parametrizado por $v c(G)$ e $v c^{+}(G)$}

Um problema de decisão em uma classe de grafos $\mathcal{C}$ é tratável com parâmetro fixo (FPT) em algum parâmetro $k=k(G)$ em tempo $O\left(n^{c}\right)$ (para uma constante $c$ ) se existe algoritmo em tempo $O\left(f(k) \cdot n^{c}\right)$ que o resolve para qualquer grafo $G$ de $\mathcal{C}$, onde $f$ é uma função que depende apenas de $k=k(G)$. Nesse caso, dizemos que o tempo é $O^{*}(f(k))$.

Em 2015, [Boria et al. 2015] obtiveram algoritmo FPT de tempo $O^{*}\left(1.5397^{v c^{+}}\right)$ para computar $v c^{+}(G)$, que pode ser usado para decidir se um grafo é bem coberto. No teorema abaixo, nós estendemos esse resultado.

Teorema 2 É possível decidir se um grafo é bem coberto em tempo $O^{*}\left(1.4656^{v c^{+}}\right)$.

Em 2015, [Boria et al. 2015] também obtiveram algoritmo FPT de tempo $O^{*}\left(2.8284^{v c}\right)$ para computar $v c^{+}(G)$. No teorema abaixo, estendemos esse resultado para um algoritmo FPT de tempo melhor $O^{*}\left(2^{v c}\right)$ que enumera todas as coberturas minimais de um grafo. 
Teorema 3 É possível enumerar todas as coberturas minimais de vértices de um grafo em tempo $O^{*}\left(2^{v c}\right)$.

Prova: [Esboço] Seja $C$ uma cobertura mínima de vértices de $G$. Logo toda aresta tem uma extremidade em $C$. Então, para toda partição de $C$ em dois conjuntos $A$ e $B$ $(A \cup B=C, A \cap B=\emptyset)$, temos que $(A \cup N(B)) \backslash B$ é uma cobertura de $G$ se não existe aresta com ambas as extremidades em $B$. Além disso, para toda cobertura minimal $C^{\prime}$ de $G, A=C \cap C^{\prime}$ e $B=C \backslash C^{\prime}$ formam uma partição de $C$ tal que $C^{\prime}=(A \cup N(B)) \backslash B$, pois $C^{\prime} \backslash C \subseteq N(B)$ (visto que $C^{\prime}$ é uma cobertura e é minimal). Portanto, podemos enumerar todas as coberturas minimais de $G$ verificando para cada partição $(A, B)$ de $C$ se $(A \cup N(B)) \backslash B$ é uma cobertura minimal de $G$. Note que é possível verificar se um conjunto é uma cobertura minimal em tempo $O(m+n)$. Como existem $2^{|C|}$ partições de $C,|C|=v c(G)$ e é possível obter uma cobertura mínima $C$ em tempo $O\left(2^{v c} \cdot(m+n)\right)$, o resultado segue.

\section{FPT parametrizado por $\alpha(G)=n-v c(G)$}

A treewidth local [Eppstein 2000] de um grafo $G$ é a função $l t w_{G}: \mathbb{N} \rightarrow \mathbb{N}$ que associa à cada $r \in \mathbb{N}$ a treewidth máxima de uma $r$-vizinhança de $G$. Ou seja, $l t w_{G}(r)=$ $\max _{v \in V(G)}\left\{t w\left(G\left[N_{r}(v)\right]\right\}\right.$, onde $N_{r}(v)$ é o conjunto de vértices à distância no máximo $r$ de $v$. Dizemos que uma classe $\mathcal{C}$ de grafos tem treewidth local limitada se existe uma função $f_{\mathcal{C}}: \mathbb{N} \rightarrow \mathbb{N}$ tal que, para todo $G \in \mathcal{C}$ e $r \in \mathbb{N}$, ltw $w_{G}(r) \leq f_{\mathcal{C}}(r)$. Sabe-se que grafos com genus limitado e grafos com grau máximo limitado tem treewidth local limitada [Eppstein 2000]. Em particular, $l t w_{G}(r) \leq \Delta(G)^{r}$ e, se $G$ é planar, ltw $w_{G}(r) \leq$ $3 r-1$ [Bodlaender 1998]. Nós provamos o seguinte:

Teorema 4 Decidir se um grafo é bem coberto é FPT parametrizado por $\alpha(G)=n-$ $v c(G)$ em tempo $O\left(n^{2}\right)$ para grafos com treewidth local limitada.

Prova: [Esboço] Seja WellCov $k$ a seguinte fórmula lógica de $1^{\circ}$-ordem que é verdadeira se e só se $G$ não tem dois conjuntos independentes $X$ e $Y \operatorname{com}|X|=k$, $|Y|=k-1$ e $Y$ sendo maximal:

$$
\begin{aligned}
\text { WellCov } & :=\forall x_{1}, \ldots, x_{k} \forall y_{1}, \ldots, y_{k-1}\left(\bigwedge_{1 \leq i<j \leq k} x_{i} \neq x_{j}\right) \wedge \operatorname{Indep}\left(\left\{x_{1}, \ldots, x_{k}\right\}\right) \\
& \rightarrow \neg\left(\operatorname{Indep}\left(\left\{y_{1}, \ldots, y_{k-1}\right\}\right) \wedge \operatorname{Maximal}\left(\left\{y_{1}, \ldots, y_{k-1}\right\}\right),\right.
\end{aligned}
$$

onde $\operatorname{Indep}(X):=\forall x, y(x \in X \wedge y \in X) \rightarrow \neg E(x, y)$ e $\operatorname{Maximal}(X):=\forall y \exists x(y \notin$ $X) \rightarrow(x \in X) \wedge E(x, y)$. Note que, se $G$ não é bem coberto, então existem conjuntos independentes $X$ e $Y$ com $2 \leq|X| \leq \alpha,|Y|=|X|-1$ e $Y$ sendo maximal. Assim, seja $W$ ellCov a fórmula lógica de $1^{\circ}$-ordem que é verdadeira se e só se $G$ é bem coberto:

$$
\text { WellCov }:=\bigwedge_{2 \leq k \leq \alpha} \text { WellCov }
$$


Como WellCov contém no máximo $\alpha^{2}$ variáveis, temos pelo Teorema de FrickGrohe (veja [Flum and Grohe 2006]) que o problema de decidir a boa cobertura é FPT parametrizado por $\alpha(G)$ em tempo $O\left(n^{2}\right)$ para grafos com treewidth local limitada.

Podemos obter algoritmos FPT específicos (parametrizados por $\alpha(G)$ ) para grafos $d$-degenerados, como grafos planares ou grau máximo limitado. Um grafo é $d$-degenerado se todo subgrafo induzido tem um vértice com grau no máximo $d$. A degenerância de $G$ é o menor $d$ tal que $G$ é $d$-degenerado. Por exemplo, grafos periplanares, grafos planares e grafos com grau máximo $\Delta$ tem degenerância no máximo 2,5 e $\Delta$, respectivamente.

Teorema 5 O problema de decidir se um grafo é bem coberto é FPT parametrizado por $\alpha=\alpha(G)=n-v c(G)$ em tempo $O\left((d+1)^{\alpha} \cdot(m+n)\right)$ para grafos d-degenerados, para todo $d>0$. Além disso, o tempo é $O\left(7^{\alpha} \cdot(m+n)\right)$, se $G$ tem genus limitado.

Prova: [Esboço] Seja $G$ um grafo $d$-degenerado. O algoritmo usa uma árvore de busca com altura $\alpha(G)$ em que cada nó tem um grafo associado. A raiz da árvore representa o grafo $G$. Uma folha é um nó com altura $\alpha(G)$ ou com grafo associado vazio. Seja $h$ um nó não-folha. Ramificamos $h$ de acordo com um vértice $v$ com grau mínimo no grafo associado de $h$. Seja $N[v]=\left\{u_{1}, \ldots, u_{\ell}\right\}$, onde $\ell=|N[v]| \leq d+1$. O nó $h$ terá $\ell+1$ filhos $h_{1}, h_{2}, \ldots, h_{\ell}$ na árvore. No nó filho $h_{i}(1 \leq i \leq \ell)$, remova $N\left[u_{i}\right]$ do grafo associado, que também é $d$-degenerado. Se existem duas folhas com diferentes alturas, retorne NÃO (pois $G$ tem conjunto independente maximal que não é máximo). Caso contrário, retorne SIM. Note que a altura da árvore é no máximo $\alpha(G)$ e cada nó tem no máximo $d+1$ filhos. Portanto, a árvore tem no máximo $(d+1)^{\alpha}$ nós e o tempo total é $O\left((d+1)^{\alpha} \cdot(m+n)\right)$, pois cada nó leva tempo $O(m+n)$.

\section{References}

Babel, L., Kloks, T., Kratochvil, J., Kratsch, D., Muller, H., and Olariu, S. (2001). Efficient algorithms for graphs with few p4's. Discrete Mathematics, 235(1):29 - 51.

Bodlaender, H. L. (1998). A partial k-arboretum of graphs with bounded treewidth. Theoretical Computer Science, 209(1):1 - 45.

Boria, N., Croce, F. D., and Paschos, V. T. (2015). On the max min vertex cover problem. Discrete Applied Mathematics, 196:62 - 71.

Caro, Y., Sebő, A., and Tarsi, M. (1996). Recognizing greedy structures. Journal of Algorithms, 20(1):137 - 156.

Eppstein, D. (2000). Diameter and treewidth in minor-closed graph families. Algorithmica, 27:275-291.

Flum, J. and Grohe, M. (2006). Parameterized Complexity Theory (Texts in Theoretical Computer Science. An EATCS Series). Springer-Verlag New York, Inc.

Giakoumakis, V. (1996). P4-laden graphs: A new class of brittle graphs. Information Processing Letters, 60(1):29 - 36.

Klein, S., de Mello, C. P., and Morgana, A. (2013). Recognizing well covered graphs of families with special p4-components. Graphs and Combinatorics, 29:553-567.

Plummer, M. D. (1970). Some covering concepts in graphs. Journal of Combinatorial Theory, 8(1):91 - 98 . 